\title{
ON THE DISCOUNTED PENALTY FUNCTION IN A MARKOV-DEPENDENT RISK MODEL
}

\author{
HANSJÖRg Albrecher $^{a, *} \quad$ OnNo J. BoxmA ${ }^{b}$ \\ a Graz University of Technology, Steyrergasse 30, A-8010 Graz, Austria \\ ${ }^{b}$ Eindhoven University of Technology and EURANDOM, P.O. Box 513, 5600 MB Eindhoven, The Netherlands
}

\begin{abstract}
We present a unified approach to the analysis of several popular models in collective risk theory. Based on the analysis of the discounted penalty function in a semi-Markovian risk model by means of Laplace-Stieltjes transforms, we rederive and extend some recent results in the field. In particular, the classical compound Poisson model, Sparre Andersen models with phase-type interclaim times and models with causal dependence of a certain Markovian type between claim sizes and interclaim times are contained as special cases.
\end{abstract}

Keywords: Dependence; classical risk model; Sparre Andersen model; time of ruin; deficit at ruin; surplus before ruin

\section{Introduction}

Let us consider the following risk model for the surplus process $R(t)$ of an insurance portfolio:

$$
R(t)=x+c t-\sum_{j=1}^{N(t)} X_{j},
$$

where $x$ is the initial capital, $c$ is the premium density which is assumed to be constant, $X_{j}$ is the size of the $j$ th claim and $N(t)$ is the number of claims up to time $t$. In classical risk theory, the claims $X_{j}$ and the claim number process $N(t)$ are assumed to be independent. However, in many applications the independence assumption is too restrictive and recently several authors looked at more general models where this assumption is relaxed in some way (see Asmussen [5] for a survey on the subject).

In this paper we will consider a semi-Markovian dependence structure of the following type: Let $W_{i}$ denote the time between the arrival of the $(i-1)$ th and the $i$ th

${ }^{*}$ Corresponding author. Email: albrecher@TUGraz. at; Supported by Fellowship F/04/009 of the K.U. Leuven and the Austrian Science Foundation Project S-8308-MAT 
claim and $W_{0}=X_{0}=0$ a.s. Then

$$
\begin{aligned}
\mathbb{P}\left(W_{n+1} \leq x, X_{n+1}\right. & \left.\leq y, Z_{n+1}=j \mid Z_{n}=i,\left(W_{r}, X_{r}, Z_{r}\right), 0 \leq r \leq n\right) \\
= & \mathbb{P}\left(W_{1} \leq x, X_{1} \leq y, Z_{1}=j \mid Z_{0}=i\right)=\left(1-e^{-\lambda_{i} x}\right) p_{i j} B_{j}(y),
\end{aligned}
$$

where $\left\{Z_{n}, n \geq 0\right\}$ is an irreducible discrete-time Markov chain with state space $\{1, \ldots, M\}$ and transition matrix $P=\left(\left(p_{i j}\right), 1 \leq i, j \leq M\right)$. Thus at each instant of a claim, the Markov chain jumps to a state $j$, and the distribution $B_{j}$ of the claim depends on the new state $j$. Then the next interarrival time is exponentially distributed with parameter $\lambda_{j}$. Note that given the states $Z_{n-1}$ and $Z_{n}$, the quantities $W_{n}$ and $X_{n}$ are independent, but there is autocorrelation among consecutive claim sizes and among consecutive interclaim times as well as cross-correlation between $W_{n}$ and $X_{n}$. This semi-Markov process was first considered in Janssen and Reinhard [18], where a formal solution for the survival probabilities in terms of an infinite series of matrix convolutions was derived.

We considerably generalize the approach in Janssen and Reinhard [18] and investigate the discounted penalty function in such a risk model by means of LaplaceStieltjes transforms (LST). This allows to obtain information on several characteristics of the ruin process.

The model considered in this paper is quite general: it contains the compound Poisson model $(M=1)$ and Sparre Andersen models with (generalized) Erlang(n)interclaim distributions (see e.g. Gerber and Shiu [16], Li and Garrido [20]) as well as phase-type interclaim distributions (see Avram and Usabel [6] and Li and Garrido [21]) as special cases (just choose appropriate transition probabilities and let $B_{j}$ be degenerate at 0 for all but one state among $\{1, \ldots, M\})$. Moreover, it also covers models with causal dependence structures of the type considered in Albrecher and Boxma [3], namely that the distribution of the inter-arrival time depends on the size of the previous claim in a specified way. To see this, choose a generic claim size random variable $X$ and for all $i=1, \ldots, M, p_{i j}=\mathbb{P}\left(X \in A_{j}\right)$ for some (possibly random) interval $A_{j} \subset \mathbb{R}$ and $B_{j} \sim X \mid X \in A_{j}$ (cf. Section 6.3). Note that by considering state-dependent transition probabilities $p_{i j}=\mathbb{P}\left(X \in A_{j} \mid\right.$ current state $i$ ), we here arrive at a more general model that also allows the claim size distribution itself to depend on the state of the Markov chain. The purpose of this paper can also be seen as to provide an umbrella to the analysis of all these risk models.

A fluid queue approach for the Laplace transform of the time until ruin (which is a special case of the discounted penalty function) in a related model can be found in Badescu et al. [7]. For an analysis of the time until ruin based on the methodology of piece-wise deterministic Markov processes, see Jacobsen [17]. For a study on the asymptotic behavior of the ruin function in the presence of dependence between interclaim times and claim sizes based on random walk techniques, see Albrecher and Teugels [4]. Adan and Kulkarni [1] recently considered a queueing model with dependence structure (2) with $\lambda_{i}$ replaced by $\lambda_{j}$. Translated to a risk model setting, the latter means that an interclaim time of state $j$ is always followed by a claim size 
of state (and thus distribution) $j$ (whereas in model (2) it is the other way round). In principle, a similar analysis can be developed for this model, too. However, in view of applications, (2) seems more appealing.

The paper is organized as follows. In Section 2, an explicit expression for the Laplace transform of the discounted penalty function in model (2) is derived. Section 3 then gives an explicit formula for the discounted penalty function for zero initial capital. In Section 4, the asymptotic behavior of the penalty function is investigated for light-tailed claim sizes. In concrete cases, it is sometimes not possible to explicitly evaluate the occurring expressions. Thus, in Section 5 it is shown how to (at least) obtain arbitrary moments of the time to ruin, surplus before ruin and the deficit at ruin. Finally, in Section 6, we specify examples and use the results of the paper to rederive and extend various formulas from the risk theory literature.

\section{An equation for the discounted penalty func- tion}

Let $\mu_{i}^{(j)}$ denote the $j$ th moment of distribution $B_{i}$, given it exists and furthermore $\mu_{i}:=\mu_{i}^{(1)}$. In the sequel we will always assume the net profit condition

$$
\sum_{i=1}^{M} \pi_{i} \mu_{i}<c \sum_{i=1}^{M} \pi_{i} \lambda_{i}^{-1},
$$

where $\pi=\left(\pi_{1}, \ldots, \pi_{M}\right)$ is the stationary distribution of $\left\{Z_{n}\right\}$. We are now interested in various characteristics of the risk model (1) together with (2). Gerber and Shiu [15] introduced the by now classical discounted penalty function at ruin

$$
m_{\delta}(x):=\mathbb{E}\left(w\left(R\left(T_{x}^{-}\right),\left|R\left(T_{x}\right)\right|\right) e^{-\delta T_{x}} 1_{\left\{T_{x}<\infty\right\}}\right)
$$

where $T_{x}$ denotes the time of ruin with initial capital $x, R\left(T_{x}^{-}\right)$is the surplus immediately before ruin, $\left|R\left(T_{x}\right)\right|$ is the deficit at ruin and the penalty $w\left(x_{1}, x_{2}\right)$ is an arbitrary non-negative function on $[0, \infty) \times[0, \infty) . \delta \geq 0$ may be interpreted as a force of interest, but (4) may also be considered in terms of a Laplace transform with $\delta$ as its argument. The function $m_{\delta}(x)$ contains a lot of useful information about the ruin process. For example, if $w \equiv 1$, then $m_{\delta}(x)$ is the LST of the time to ruin given it occurs, and $m_{0}(x)$ is then simply the ruin probability $\psi(x)$. For $w\left(x_{1}, x_{2}\right)=1_{\left\{x_{1} \leq y_{1}\right\}} 1_{\left\{x_{2} \leq y_{2}\right\}}, m_{0}(x)$ is just the joint distribution of the surplus before ruin and the deficit at ruin.

We will now derive an integro-differential equation for $m_{\delta}(x)$ for our Markov additive risk process. Let $m_{\delta, i}(x)$ denote the discounted penalty function given that $Z_{0}=i$. 
Then by conditioning on the time interval $(0, d t)$, we obtain

$$
\begin{aligned}
& m_{i}(x)=\left(1-\lambda_{i} d t\right) e^{-\delta d t} m_{\delta, i}(x+c d t)+\lambda_{i} d t \sum_{j=1}^{M} p_{i j} \int_{0}^{x+c d t} e^{-\delta d t} m_{\delta, j}(x+c d t-y) d B_{j}(y) \\
& +\lambda_{i} d t \sum_{j=1}^{M} p_{i j} \int_{x+c d t}^{\infty} e^{-\delta d t} w(x+c d t, y-x-c d t) d B_{j}(y)+o(d t) \quad(i=1, \ldots, M) .
\end{aligned}
$$

Taylor expansion and rearranging yields

$$
\begin{aligned}
c \frac{d m_{\delta, i}}{d x}(x)-\left(\lambda_{i}+\delta\right) & m_{\delta, i}(x)+\lambda_{i} \sum_{j=1}^{M} p_{i j} \int_{0}^{x} m_{\delta, j}(x-y) d B_{j}(y) \\
& +\lambda_{i} \sum_{j=1}^{M} p_{i j} \int_{x}^{\infty} w(x, y-x) d B_{j}(y)=0 \quad(i=1, \ldots, M) .
\end{aligned}
$$

Define, for Re $s \geq 0$ the Laplace(-Stieltjes) transforms

$$
\begin{aligned}
\tilde{m}_{\delta, i}(s) & :=\int_{0}^{\infty} e^{-s x} m_{\delta, i}(x) d x \\
\tilde{b}_{i}(s) & :=\int_{x=0}^{\infty} e^{-s x} d B_{i}(x), \quad(i=1, \ldots, M), \\
\tilde{\omega}_{i}(s) & :=\int_{x=0}^{\infty} e^{-s x} \int_{x}^{\infty} w(x, y-x) d B_{i}(y) d x, \quad(i=1, \ldots, M) .
\end{aligned}
$$

Then we obtain for $i=1, \ldots, M$,

$$
\operatorname{cs} \tilde{m}_{\delta, i}(s)-c m_{\delta, i}(0)-\left(\lambda_{i}+\delta\right) \tilde{m}_{\delta, i}(s)+\lambda_{i} \sum_{j=1}^{M} p_{i j}\left(\tilde{m}_{\delta, j}(s) \tilde{b}_{j}(s)+\tilde{\omega}_{j}(s)\right)=0,
$$

or in matrix notation,

$$
((c s-\delta) I-\Lambda+\Lambda P \tilde{B}(s)) \overrightarrow{\tilde{m}}_{\delta}(s)=c \vec{m}_{\delta}(0)-\Lambda P \overrightarrow{\tilde{\omega}}(s),
$$

where $I$ is the identity matrix, $\Lambda=\operatorname{diag}\left(\lambda_{1}, \ldots, \lambda_{M}\right), \tilde{B}(s)=\operatorname{diag}\left(\tilde{b}_{1}(s), \ldots, \tilde{b}_{M}(s)\right)$ and $\overrightarrow{\tilde{m}}_{\delta}(s)=\left(\tilde{m}_{\delta, 1}(s), \ldots, \tilde{m}_{\delta, M}(s)\right)$.

Thus it remains to solve a system of linear equations. First, the quantities $m_{\delta, i}(0)$ have to be determined. For that purpose, denote

$$
A_{\delta}(s):=(c s-\delta) I-\Lambda+\Lambda P \tilde{B}(s)
$$

Proposition 2.1. (i) The equation $\operatorname{det}\left(A_{0}(s)\right)=0$ has one zero $s_{1}=0$ and $M-1$ zeroes $s_{2}, \ldots, s_{M}$ with $\operatorname{Re}\left(s_{i}\right)>0$.

(ii) If $\delta>0$ then $\operatorname{det}\left(A_{\delta}(s)\right)=0$ has $M$ zeroes $s_{1}, \ldots, s_{M}$ with $\operatorname{Re}\left(s_{i}\right)>0$. 
Proof: Case (i): If $\delta=0$, then the statement immediately follows from Theorem 3.2 of [1] (after transposing their matrix).

Case (ii): We follow an idea in [9], see also [1]. Let $C$ denote a circle with its center at $\left[\delta+\max _{1 \leq i \leq M} \lambda_{i}\right] / c$ and radius $\left[\delta+\max _{1 \leq i \leq M} \lambda_{i}\right] / c$, and let $A_{\delta}(s, u):=$ $(c s-\delta) I-\Lambda+u \Lambda P \tilde{B}(s), 0 \leq u \leq 1$. We first prove, for $0 \leq u \leq 1$, that

$$
\operatorname{det}\left(A_{\delta}(s, u)\right) \neq 0 \text { for } s \in C \text {. }
$$

The matrix $A_{\delta}(s, u)$ is diagonally dominant for $0 \leq u \leq 1$, since

$$
\begin{aligned}
& \left|c s-\delta-\lambda_{i}+u \lambda_{i} p_{i, i} \tilde{b}_{i}(s)\right| \geq\left|\delta+\lambda_{i}-c s\right|-\left|u \lambda_{i} p_{i, i} \tilde{b}_{i}(s)\right| \\
\geq & \delta+\lambda_{i}-u \lambda_{i} p_{i, i} \tilde{b}_{i}(0)>u \lambda_{i}\left(1-p_{i, i} \tilde{b}_{i}(0)\right) \\
= & u \lambda_{i} \sum_{j \neq i} p_{i, j} \tilde{b}_{j}(0) \geq\left|u \lambda_{i} \sum_{j \neq i} p_{i, j} \tilde{b}_{j}(s)\right| .
\end{aligned}
$$

The diagonal dominance implies (cf. [23, pp.146-147]) that $\operatorname{det} A_{\delta}(s, u) \neq 0$ for $s \in C$.

Now let $f(u)$ denote the number of zeroes of $\operatorname{det}\left(A_{\delta}(s, u)\right)$ in $C^{+}$, the interior of $C$. Then

$$
f(u)=\frac{1}{2 \pi i} \int_{C} \frac{\frac{d}{d s} \operatorname{det}\left(A_{\delta}(s, u)\right)}{\operatorname{det}\left(A_{\delta}(s, u)\right)} \mathrm{d} s .
$$

Hence $f(u)$ is a continuous function on $[0,1]$, integer valued, and therefore constant. $f(0)=M$, because $\operatorname{det}\left(A_{0}(s)\right)=\operatorname{det}((c s-\delta) I-\Lambda)=0$ for $s_{i}^{*}:=\frac{\delta+\lambda_{i}}{c}, 1 \leq i \leq M$. Hence also $f(1)=M$.

Remark 2.1. It follows from the above proof for Case (ii), and from Remark 6.2 of [1] for Case (i), that the zeroes are located in the interior $C^{+}\left(s_{1}=0\right.$ is located on $C$ in Case (i)).

Remark 2.2. The solutions of

$$
\operatorname{det}\left(A_{\delta}(s)\right)=0
$$

are intimately connected with the behavior of $m_{\delta}(x)$ : zeroes with negative real part determine the asymptotic behavior, whereas zeroes in the right half-plane determine the constants in the exact expressions for $m_{\delta}(x)$. Since for $M=1$, this equation is known as the Lundberg fundamental equation (see e.g. [16]), we call (10) the generalized Lundberg fundamental equation.

Now, under the assumption that the functions $m_{\delta, i}(x)$ do not grow super-exponentially fast (which is fulfilled for all penalty functions of practical interest), $\tilde{m}_{\delta, i}(s)$ are analytic functions for $\operatorname{Re}(s) \geq 0$, so that for each of the $M$ zeroes $s_{1}, \ldots, s_{M}$ we can proceed in the following way: Determine a non-trivial solution $\vec{k}_{i}$ of

$$
A_{\delta}^{T}\left(s_{i}\right) \vec{k}_{i}=\overrightarrow{0}
$$


for each $i=1, \ldots, M$. Since we then have

$$
0=\overrightarrow{\tilde{m}}_{\delta}\left(s_{i}\right)^{T} A_{\delta}^{T}\left(s_{i}\right) \vec{k}_{i}=\left(c \vec{m}_{\delta}(0)-\Lambda P \overrightarrow{\tilde{\omega}}\left(s_{i}\right)\right)^{T} \vec{k}_{i},
$$

this gives $\mathrm{M}$ linear equations for $m_{\delta, 1}(0), \ldots, m_{\delta, M}(0)$.

Remark 2.3. For $\delta=0$, the zeroes $s_{1}, \ldots, s_{M}$ can always be obtained numerically. Moreover, if the involved claim size distributions have a rational Laplace transform, then the discounted penalty function can be obtained explicitly by inversion of the Laplace transform of the solution of $(7)$.

\section{Zero initial capital}

The following explicit expression for the discounted penalty function with zero initial capital can be obtained:

Proposition 3.1. Let $K:=\left(\vec{k}_{1}, \ldots, \vec{k}_{M}\right)^{T}$ and let $\operatorname{det} K_{j_{2}, i}$ denote the minor of $K$ with respect to row $j_{2}$ and column $i$. Then

$$
m_{\delta, i}(0)=\sum_{j_{1}=1}^{M} \sum_{j_{2}=1}^{M} C_{j_{1}, j_{2}}^{(i)}\left(s_{1}, \ldots, s_{M}, \delta\right) \tilde{\omega}_{j_{1}}\left(s_{j_{2}}\right), \quad(i=1, \ldots, M)
$$

where the coefficients $C_{j_{1}, j_{2}}^{(i)}\left(j_{1}, j_{2}=1, \ldots, M\right)$ are given by

$$
C_{j_{1}, j_{2}}^{(i)}=\frac{(-1)^{i+j_{2}} \cdot \operatorname{det} K_{j_{2}, i} \cdot \sum_{l=1}^{M} \lambda_{l} p_{l, j_{1}} k_{j_{2}, l}}{c \operatorname{det} K}
$$

with $k_{j_{2}, l}$ denoting the $l$-th component of vector $\vec{k}_{j_{2}}(l=1, \ldots, M)$.

Proof: Equations (11) can be written as

$$
c K \vec{m}_{\delta}(0)=\left(\begin{array}{c}
\sum_{j_{1}=1}^{M} d_{j_{1}}^{(1)} \tilde{\omega}_{j_{1}}\left(s_{1}\right) \\
\vdots \\
\sum_{j_{1}=1}^{M} d_{j_{1}}^{(M)} \tilde{\omega}_{j_{1}}\left(s_{M}\right)
\end{array}\right)
$$

with $d_{j_{1}}^{\left(j_{2}\right)}=\sum_{l=1}^{M} \lambda_{l} p_{l, j_{1}} k_{j_{2}, l}$. An application of Cramér's rule gives

$$
m_{\delta, i}(0)=\frac{\left|\begin{array}{ccccccc}
k_{1,1} & \cdots & k_{1, i-1} & \sum_{j_{1}=1}^{M} d_{j_{1}}^{(1)} \tilde{\omega}_{j_{1}}\left(s_{1}\right) & k_{1, i+1} & \cdots & k_{1, M} \\
\vdots & & \vdots & \vdots & \vdots & & \vdots \\
k_{M, 1} & \cdots & k_{M, i-1} & \sum_{j_{1}=1}^{M} d_{j_{1}}^{(M)} \tilde{\omega}_{j_{1}}\left(s_{M}\right) & k_{M, i+1} & \cdots & k_{M, M}
\end{array}\right|}{c \operatorname{det} K} .
$$

Expanding the determinant in the numerator along the $i$-th column then yields the desired result. 
Let $f_{i}\left(y_{1}, y_{2}, t \mid x\right)$ denote the (defective) joint density function of $R\left(T_{x}^{-}\right),\left|R\left(T_{x}\right)\right|$ and $T_{x}$ given $Z_{0}=i$, i.e.

$$
m_{\delta, i}(x)=\int_{y_{1}=0}^{\infty} \int_{y_{2}=0}^{\infty} \int_{t=0}^{\infty} w\left(y_{1}, y_{2}\right) e^{-\delta t} f_{i}\left(y_{1}, y_{2}, t \mid x\right) d t d y_{2} d y_{1}
$$

and define the discounted joint density function of surplus prior to and after ruin by $f_{i}\left(y_{1}, y_{2} \mid x\right)=\int_{0}^{\infty} e^{-\delta t} f_{i}\left(y_{1}, y_{2}, t \mid x\right) d t$. The following result generalizes Formula (8.3) of Gerber and Shiu [16]:

Corollary 3.2. Assume that the claim size distributions $B_{i}(i=1, \ldots, M)$ are absolutely continuous with density function $b_{i}(y)$. Then

$$
f_{i}\left(y_{1}, y_{2} \mid 0\right)=\sum_{j_{1}=1}^{M} \sum_{j_{2}=1}^{M} C_{j_{1}, j_{2}}^{(i)}\left(s_{1}, \ldots, s_{M}, \delta\right) e^{-s_{j_{2}} y_{1}} b_{j_{1}}\left(y_{1}+y_{2}\right), \quad(i=1, \ldots, M)
$$

Proof: Choose $w\left(x_{1}, x_{2}\right)$ to be the Dirac delta function with respect to $x_{1}=y_{1}, x_{2}=$ $y_{2}$ (i.e. $\left.\tilde{\omega}_{i}(s)=e^{-s y_{1}} b_{i}\left(y_{1}+y_{2}\right)\right)$. Then the assertion is a direct consequence of Proposition 3.1.

Accordingly, we obtain for the discounted marginal density of the surplus prior to ruin

$$
f_{i}\left(y_{1} \mid 0\right)=\int_{0}^{\infty} f_{i}\left(y_{1}, y_{2} \mid 0\right) d y_{2}=\sum_{j_{1}=1}^{M} \sum_{j_{2}=1}^{M} C_{j_{1}, j_{2}}^{(i)}\left(s_{1}, \ldots, s_{M}, \delta\right) e^{-s_{j_{2}} y_{1}}\left(1-B_{j_{1}}\left(y_{1}\right)\right)
$$

and for the discounted marginal density $f_{i}\left(y_{2} \mid 0\right)=\int_{0}^{\infty} f_{i}\left(y_{1}, y_{2} \mid 0\right) d y_{1}$ of the deficit at ruin

$$
f_{i}\left(y_{2} \mid 0\right)=\sum_{j_{1}=1}^{M} \sum_{j_{2}=1}^{M} C_{j_{1}, j_{2}}^{(i)}\left(s_{1}, \ldots, s_{M}, \delta\right) e^{s_{j_{2}} y_{2}}\left(\tilde{b}_{j_{1}}\left(s_{j_{2}}\right)-\int_{0}^{y_{2}} e^{-s_{j_{2}} z} b_{j_{1}}(z) d z\right) .
$$

\section{Asymptotic behavior}

From Section 2 it follows that

$$
\overrightarrow{\tilde{m}}_{\delta}(s)=\frac{A_{\delta, a d j}(s)\left(c \vec{m}_{\delta}(0)-\Lambda P \overrightarrow{\tilde{\omega}}(s)\right)}{\operatorname{det} A_{\delta}(s)}
$$

is a vector of analytic functions for $\operatorname{Re}(s)>0$ (here $A_{\delta, \text { adj }}(s)$ denotes the adjunct matrix of $A_{\delta}(s)$ ).

Let us assume that all the $\operatorname{LST} \tilde{b}_{i}(s)$ of the claim size distributions $B_{i}$ exist in a neighborhood of the origin. Then, due to the structure of (15), the functions $\tilde{m}_{\delta, i}(s)$ are analytic for all $s$ with $R e(s)>-R_{\delta}$, where $-R_{\delta}$ denotes the zero with largest real part in the negative halfplane of $\operatorname{det} A_{\delta}(s)$ (which is the generalized Lundberg 
adjustment coefficient). From the damping property of Laplace transforms, we have $\mathcal{L}\left(e^{R_{\delta} x} m_{\delta, i}(x)\right)=\tilde{m}_{\delta, i}\left(s-R_{\delta}\right)$ so that

$$
\lim _{x \rightarrow \infty} e^{R_{\delta} x} \vec{m}_{\delta}(x)=\lim _{s \rightarrow 0} s \overrightarrow{\tilde{m}}_{\delta}\left(s-R_{\delta}\right)=\vec{C},
$$

given that the limit exists (which for instance is guaranteed if $e^{R_{\delta} x} m_{\delta, i}(x)$ is monotonically increasing in $x$ for each $i=1, \ldots, M$, see e.g. Doetsch [14]).

For convenience, let $s=-R_{\delta}$ be a simple pole of $\tilde{m}_{\delta, i}(s)$, then we obtain, using de L'Hospital:

$$
\vec{C}=\frac{A_{\delta, a d j}\left(-R_{\delta}\right)\left(c \vec{m}_{\delta}(0)-\Lambda P \overrightarrow{\tilde{\omega}}\left(-R_{\delta}\right)\right)}{\left.\frac{\partial}{\partial s}\left(\operatorname{det} A_{\delta}(s)\right)\right|_{s=-R_{\delta}}} .
$$

Thus the discounted penalty function decays exponentially with initial capital $x$ at rate $R_{\delta}$ and the corresponding constants are given by (16).

\section{Moments of three Characteristics of the Ruin Process}

\subsection{Moments of the Time to Ruin}

First take $w(\cdot, \cdot) \equiv 1$ and define $f_{n, i}(s):=\left.\frac{\partial^{n} \tilde{m}_{\delta, i}(s)}{\partial \delta^{n}}\right|_{\delta=0}$ which is (up to the sign) the Laplace transform w.r.t. $x$ of the $n$th moment of the time to ruin. Differentiation of Formula (6) and substitution of $\delta=0$ gives after some algebraic manipulations:

$$
\left(c s-\lambda_{i}\right) f_{n, i}(s)-n f_{n-1, i}(s)+\lambda_{i} \sum_{j=1}^{M} p_{i j} \tilde{b}_{j}(s) f_{n, j}(s)=\left.c \frac{\partial^{n} m_{\delta, i}(0)}{\partial \delta^{n}}\right|_{\delta=0},
$$

or in matrix form

$$
A_{0}(s) \vec{f}_{n}(s)=\left.c \frac{\partial^{n} \vec{m}_{\delta}(0)}{\partial \delta^{n}}\right|_{\delta=0}+n \vec{f}_{n-1}(s) .
$$

Note that $\vec{f}_{0}(s)=\overrightarrow{\tilde{m}}_{0}(s)=\overrightarrow{\tilde{\psi}}(s)$, where $\tilde{\psi}_{i}(s)$ is the Laplace transform of the ruin probability $\psi_{i}(x)(i=1, \ldots, M)$. The vector $\overrightarrow{\tilde{\psi}}(s)$ is available as the solution of $(6)$ for $\delta=0$. Thus we get a recursion for the $n$th moment of the time to ruin. In the $j$ th recursive step, we have to determine the $M$ constants $\left.\frac{\partial^{j} m_{\delta, i}(0)}{\partial \delta^{j}}\right|_{\delta=0}(i=1, \ldots, M)$ in the above way using the $M$ zeroes of $\operatorname{det} A_{0}(s)=0$ in the positive halfplane (which can always be obtained numerically).

\subsection{Moments of the Surplus prior to Ruin}

Next take $w(x, y) \equiv \mathrm{e}^{-a x}$ so that $\tilde{\omega}_{i}(s)=\frac{1-\tilde{b}_{i}(s+a)}{s+a}$. Let furthermore $\delta=0$ and denote $g_{n, i}(s)=\left.\frac{\partial^{n} \tilde{m}_{0, i}(s)}{\partial a^{n}}\right|_{a=0}$, which is (up to the sign) the Laplace transform w.r.t. 
$x$ of the $n$th moment of the surplus prior to ruin. Differentiation of (6) w.r.t. $a$ and substitution of $a=0$ gives

$$
A_{0}(s) \vec{g}_{n}(s)=\left.c \frac{\partial^{n} \vec{m}_{0}(0)}{\partial a^{n}}\right|_{a=0}-\Lambda P \operatorname{diag}\left(\xi_{n, 1}(s), \ldots, \xi_{n, M}(s)\right),
$$

where

$$
\xi_{n, i}(s):=\left.\frac{\partial^{n} \tilde{\omega}_{i}(s)}{\partial a^{n}}\right|_{a=0}=\frac{(-1)^{n} n !}{s^{n+1}}\left(1-\tilde{b}_{i}(s)-\sum_{j=1}^{n} \frac{(-s)^{j}}{j !} \frac{\partial^{j} \tilde{b}_{i}(s)}{\partial s^{j}}\right) .
$$

Thus the Laplace transform of the $n$-th moment can be obtained by first determining the $M$ constants $\left.\frac{\partial^{n} m_{0, i}(0)}{\partial a^{n}}\right|_{a=0}$ using the zeroes of the generalized Lundberg's fundamental equation in the positive halfplane in the usual way and then solving the above linear system of equations.

\subsection{Moments of the Deficit at Ruin}

Finally take $w(x, y) \equiv \mathrm{e}^{-a y}$ and thus $\tilde{\omega}_{i}(s)=\frac{\tilde{b}_{i}(a)-\tilde{b}_{i}(s)}{s-a}$. Define $k_{n, i}(s):=\left.\frac{\partial^{n} \tilde{m}_{0, i}(s)}{\partial a^{n}}\right|_{a=0}$, which is (up to the sign) the Laplace transform w.r.t. $x$ of the $n$th moment of the deficit at ruin. Differentiation of (6) w.r.t. $a$ and substitution of $a=0$ gives

$$
A_{0}(s) \vec{k}_{n}(s)=\left.c \frac{\partial^{n} \vec{m}_{0}(0)}{\partial a^{n}}\right|_{a=0}-\Lambda P \operatorname{diag}\left(\eta_{n, 1}(s), \ldots, \eta_{n, M}(s)\right),
$$

where

$$
\eta_{n, i}(s):=\left.\frac{\partial^{n} \tilde{\omega}_{i}(s)}{\partial a^{n}}\right|_{a=0}=-\frac{n !}{s^{n+1}}\left(\tilde{b}_{i}(s)-\sum_{j=0}^{n} \frac{(-s)^{j}}{j !} \mathbb{E}\left(B_{i}^{j}\right)\right) .
$$

Thus the Laplace transform of the $n$-th moment can again be obtained by first determining the $M$ constants $\left.\frac{\partial^{n} m_{0, i}(0)}{\partial a^{n}}\right|_{a=0}$ using the zeroes in the positive halfplane of Lundberg's fundamental equation and subsequently solving the above linear system of equations.

\section{Examples}

\subsection{The classical compound Poisson model}

For $M=1$ we retain the classical compound Poisson risk model, and indeed from (6) it follows that in this case

$$
\tilde{m}_{\delta}(s)=\frac{c m_{\delta}(0)-\lambda \tilde{\omega}(s)}{c s-\delta-\lambda+\lambda \tilde{b}(s)}=\frac{\lambda\left(\tilde{\omega}\left(s_{1}\right)-\tilde{\omega}(s)\right)}{c s-\delta-\lambda+\lambda \tilde{b}(s)},
$$

in agreement with [11]. If the LST of the claim size distribution $B$ exists in a neighborhood of the origin, then one obtains from (16)

$$
\lim _{x \rightarrow \infty} e^{R_{\delta} x} m_{\delta}(x)=\frac{\lambda\left(\tilde{\omega}\left(s_{1}\right)-\tilde{\omega}\left(-R_{\delta}\right)\right)}{c+\lambda \tilde{b}^{\prime}\left(-R_{\delta}\right)},
$$


where $-R_{\delta}$ denotes the negative zero of $c s-\delta-\lambda+\lambda \tilde{b}(s)=0$ (which is unique, cf. [15]). In the special case $w \equiv 1$ and $\delta=0$ we have $s_{1}=0$ and $\tilde{\omega}(s)=(1-\tilde{b}(s)) / s$, so that (19) reduces to the Cramér-Lundberg approximation $\lim _{x \rightarrow \infty} e^{R_{0} x} \psi(x)=$ $\frac{\lambda \mu-c}{c+\lambda \tilde{b}^{\prime}\left(-R_{0}\right)}$.

Let us now look at moments of the time to ruin in the classical risk model and assume that $\mu^{(2)}<\infty$. Let, for $n \in \mathbb{N}, \psi_{n}(x):=\mathbb{E}\left(T_{x}^{n} 1_{\left\{T_{x}<\infty\right\}}\right)$ and $\psi_{0}(x):=\psi(x)$, the ruin probability. Then $\mathbb{E}\left(T_{x}^{n} \mid T_{x}<\infty\right)=\frac{\psi_{n}(x)}{\psi(x)}$ and the Laplace transform of $\psi_{n}(x)$ is just $(-1)^{n} f_{n}(s)$ defined in Section 5.1. Equation (17) here translates into

$$
f_{n}(s)=\frac{\left.c \frac{\partial^{n} m_{\delta}(0)}{\partial \delta^{n}}\right|_{\delta=0}+n f_{n-1}(s)}{c s-\lambda+\lambda \tilde{b}(s)} .
$$

Lemma 6.1. For the classical compound Poisson model and $w \equiv 1$, the following recursive relation holds for $n \geq 1(n \in \mathbb{N})$ :

$$
\left.\frac{\partial^{n} m_{\delta}(0)}{\partial \delta^{n}}\right|_{\delta=0}=\frac{(-1)^{n} n}{c} \int_{0}^{\infty} \psi_{n-1}(u) d u .
$$

Proof: (20) is an analytic function for $\operatorname{Re}(s) \geq 0$. Since $s=0$ is the only zero of the denominator in the positive halfplane, it follows that

$$
\begin{aligned}
&\left.\frac{\partial^{n} m_{\delta}(0)}{\partial \delta^{n}}\right|_{\delta=0}=-\frac{n}{c} \lim _{s \rightarrow 0}\left.\frac{\partial^{n-1} \tilde{m}_{\delta}(s)}{\partial \delta^{n-1}}\right|_{\delta=0} \\
&=-\left.\frac{n}{c} \int_{0}^{\infty} \frac{\partial^{n-1} m_{\delta}(u)}{\partial \delta^{n-1}}\right|_{\delta=0} d u=\frac{(-1)^{n} n}{c} \int_{0}^{\infty} \psi_{n-1}(u) d u .
\end{aligned}
$$

Using the Pollaczek-Khintchine formula $\tilde{\psi}(s)=\frac{1}{s}-\frac{c-\lambda \mu}{c s-\lambda+\lambda \tilde{b}(s)}$ and Lemma 6.1, (20) now yields

$$
f_{n}(s)=(-1)^{n} \tilde{\psi}_{n}(s)=(-1)^{n} n\left(\int_{0}^{\infty} \psi_{n-1}(u) d u+n \tilde{\psi}_{n-1}(s)\right) \frac{\frac{1}{s}-\tilde{\psi}(s)}{c-\lambda \mu}
$$

leading to

$\psi_{n}(x)=\frac{n}{c-\lambda \mu}\left(\int_{0}^{x} \psi(x-u) \psi_{n-1}(u) d u+\int_{x}^{\infty} \psi_{n-1}(u) d u-\psi(x) \int_{0}^{\infty} \psi_{n-1}(u) d u\right)$,

which is equivalent to Formula (6.29) of Lin and Willmot [22], where the result was obtained using compound geometric tails. Note that the above derivation is particularly simple.

Using the identity $\int_{0}^{\infty} \psi(x) d x=\frac{\lambda \mu^{(2)}}{2(c-\lambda \mu)}$ (which itself is a direct consequence of the Pollaczek-Khintchine formula for $\left.\mu^{(2)}<\infty\right)$, one obtains for the specific case $n=1$

$$
\mathbb{E}\left(T_{x} \mid T_{x}<\infty\right)=\frac{\int_{0}^{x} \psi(x-u) \psi(u) d u+\int_{x}^{\infty} \psi(u) d u-\frac{\lambda \mu^{(2)}}{2(c-\lambda \mu)} \psi(x)}{(c-\lambda \mu) \psi(x)},
$$


which is Formula (6.23) of Lin and Willmot [22].

Let us now choose $\delta=0$, then $s_{1}=0$ and it follows from (18) and the PollaczekKhintchine formula

$$
\tilde{m}_{0}(s)=\lambda(\tilde{\omega}(0)-\tilde{\omega}(s)) \frac{\frac{1}{s}-\tilde{\psi}(s)}{c-\lambda \mu}
$$

so that

$$
m_{0}(x)=\frac{\lambda}{c-\lambda \mu}\left(\tilde{\omega}(0)(1-\psi(x))-\int_{0}^{x}(1-\psi(x-u)) \int_{u}^{\infty} w(u, y-u) d B(y) d u\right) .
$$

The latter formula gives rise to a number of nice identities. For instance, the LST of the surplus prior to ruin is obtained for $w \equiv e^{-a x}$, i.e. $\tilde{\omega}(s)=\frac{1-\tilde{b}(a+s)}{a+s}$, and from $(21)$

$$
\mathbb{E}\left(e^{-a R_{T_{x}}^{-}} 1_{\left\{T_{x}<\infty\right\}}\right)=\frac{\lambda}{c-\lambda \mu}\left(\frac{1-\tilde{b}(a)}{a}(1-\psi(x))-\int_{0}^{x}(1-\psi(x-u)) e^{-a u} \bar{B}(u) d u\right) .
$$

This leads to the defective density of the surplus prior to ruin

$$
f\left(y_{1} \mid x\right)=\frac{\lambda}{c-\lambda \mu}\left(\bar{B}\left(y_{1}\right)(1-\psi(x))-1_{\left\{y_{1}<x\right\}} \bar{B}\left(y_{1}\right)\left(1-\psi\left(x-y_{1}\right)\right)\right),
$$

which already appeared in Dickson [10]. By differentiation of (22) we immediately obtain

$\mathbb{E}\left(\left(R_{T_{x}}^{-}\right)^{n} \mid T_{x}<\infty\right)=\frac{\lambda}{(c-\lambda \mu) \psi(x)}\left(\frac{\mu^{(n+1)}(1-\psi(x))}{n+1}-\int_{0}^{x} u^{n}(1-\psi(x-u)) \bar{B}(u) d u\right)$,

in agreement with (5.3) of Lin and Willmot [22] (again, our Laplace transform approach leads to the result in a straight-forward way).

On the other hand, the choice $w \equiv e^{-a y}$ (i.e. $\left.\tilde{\omega}(s)=\frac{\tilde{b}(a)-\tilde{b}(s)}{s-a}\right)$ in $(21)$ leads to the LST of the deficit at ruin

$$
\begin{aligned}
\mathbb{E}\left(e^{-a\left|R_{T_{x}}\right|} 1_{\left\{T_{x}<\infty\right\}}\right)=\frac{\lambda}{c-\lambda \mu}\left(\frac{1-\tilde{b}(a)}{a}(1-\psi(x))\right. \\
\left.\quad-\int_{0}^{x}(1-\psi(x-u)) \int_{u}^{\infty} e^{-a(y-u)} d B(y) d u\right)
\end{aligned}
$$

and differentiation gives the moments

$$
\begin{aligned}
\mathbb{E}\left(\left|R_{T_{x}}\right|^{n} \mid T_{x}<\infty\right)=\frac{\lambda}{(c-\lambda \mu) \psi(x)} & \frac{\mu^{(n+1)}(1-\psi(x))}{n+1} \\
& \left.-\int_{0}^{x}(1-\psi(x-u)) \int_{u}^{\infty}(y-u)^{n} d B(y) d u\right),
\end{aligned}
$$

which is another way of writing Equation (4.5) in [22].

In particular, it follows from (22) and (23) that for $x=0$ the distributions of the surplus prior to ruin and of the deficit at ruin coincide (a fact that seems to be unmentioned in the literature on the classical model). 


\subsection{Renewal models}

\subsubsection{Generalized Erlang(n)-interclaim times}

Let us assume that we start in state 1 and that

$$
P=\left(\begin{array}{ccccc}
0 & 1 & 0 & \cdots & 0 \\
0 & 0 & 1 & \cdots & 0 \\
\vdots & \vdots & \ddots & & \vdots \\
0 & 0 & 0 & \cdots & 1 \\
1 & 0 & 0 & \cdots & 0
\end{array}\right)
$$

Assume furthermore that a claim can only occur in state 1 (with claim size distribution $B_{1}=B$ and LST $\tilde{b}$ ), and the claim size distributions $B_{2}, \ldots, B_{M}$ of all other states are degenerate at zero, i.e. $\tilde{B}(s)=\operatorname{diag}(\tilde{b}(s), 1, \ldots, 1)$ and $\overrightarrow{\tilde{\omega}}(s)=$ $(\tilde{\omega}(s), 0, \ldots, 0)^{T}$.

Then $m_{\delta}(x):=m_{\delta, 1}(x)$ is the discounted penalty function for a renewal model with generalized Erlang $(\mathrm{n})$ interclaim times. Here $A_{\delta}(s)$ has the simple form

$$
A_{\delta}(s)=\left(\begin{array}{cccccc}
c s-\delta-\lambda_{1} & \lambda_{1} & 0 & \cdots & 0 & 0 \\
0 & c s-\delta-\lambda_{2} & \lambda_{2} & \cdots & 0 & 0 \\
\vdots & 0 & \ddots & \ddots & \vdots & \vdots \\
\vdots & \vdots & \cdots & c s-\delta-\lambda_{n-2} & \lambda_{n-2} & 0 \\
0 & 0 & \cdots & 0 & c s-\delta-\lambda_{n-1} & \lambda_{n-1} \\
\lambda_{n} \tilde{b}(s) & 0 & \cdots & \cdots & 0 & c s-\delta-\lambda_{n}
\end{array}\right)
$$

so that its determinant is easily calculated yielding

$$
\operatorname{det} A_{\delta}(s)=(-1)^{n}\left(\prod_{j=1}^{n}\left(\lambda_{j}+\delta-c s\right)-\tilde{b}(s) \prod_{j=1}^{n} \lambda_{j}\right) .
$$

Due to the simple transition matrix $P$ of this example, it follows from $(5)$ that $m_{\delta}(x)$ is the solution of the integro-differential equation

$$
\prod_{j=1}^{n}\left(1+\frac{\delta-c D}{\lambda_{j}}\right) m_{\delta}(x)=\int_{0}^{x} m_{\delta}(x-y) d B(y)+\int_{x}^{\infty} w(x, y-x) d B(y),
$$

where $D$ denotes the differentiation operator w.r.t. $x$.

This model has recently been studied in detail by Gerber and Shiu [16] and for $\lambda_{1}=\ldots=\lambda_{M}$ by Li and Garrido [20] (see [13, 24, 8] for the special case $n=2$ ).

Again, our formalism can be used to rederive results for this model in a quite transparent way. For instance, $m_{\delta}(0)$ follows from Proposition 3.1: Since we start in state $i=1$ and only in state 1 there is a non-degenerate claim size distribution, (12) simplifies to

$$
m_{\delta}(0)=\sum_{j_{2}=1}^{n} C_{1, j_{2}}^{(1)}\left(s_{1}, \ldots, s_{n}, \delta\right) \tilde{\omega}\left(s_{j_{2}}\right)
$$


It remains to determine the constants

$$
C_{1, j_{2}}^{(1)}\left(s_{1}, \ldots, s_{n}, \delta\right)=\frac{(-1)^{1+j_{2}} \lambda_{n} k_{j_{2}, n} \operatorname{det} K_{j_{2}, 1}}{c \operatorname{det} K} .
$$

From (11) one obtains

$$
K=\left(\begin{array}{ccccc}
\prod_{j=2}^{n} \frac{\lambda_{j}+\delta-c s_{1}}{\lambda_{j-1}} & \prod_{j=3}^{n} \frac{\lambda_{j}+\delta-c s_{1}}{\lambda_{j-1}} & \cdots & \frac{\lambda_{n}+\delta-c s_{1}}{\lambda_{n-1}} & 1 \\
\vdots & \vdots & \ddots & \vdots & \vdots \\
\prod_{j=2}^{n} \frac{\lambda_{j}+\delta-c s_{n}}{\lambda_{j-1}} & \prod_{j=3}^{n} \frac{\lambda_{j}+\delta-c s_{n}}{\lambda_{j-1}} & \cdots & \frac{\lambda_{n}+\delta-c s_{n}}{\lambda_{n-1}} & 1
\end{array}\right)
$$

leading to

$$
\operatorname{det} K=\frac{c^{\frac{n(n-1)}{2}}}{\lambda_{1} \lambda_{2}^{2} \cdots \lambda_{n-1}^{n-1}}\left|\begin{array}{cccc}
1 & s_{1} & \cdots & s_{1}^{n-1} \\
\vdots & \vdots & \ddots & \vdots \\
1 & s_{n} & \cdots & s_{n}^{n-1}
\end{array}\right|=\frac{c^{\frac{n(n-1)}{2}}}{\lambda_{1} \lambda_{2}^{2} \cdots \lambda_{n-1}^{n-1}} \prod_{\substack{j, k=1 \\
k>j}}^{n}\left(s_{k}-s_{j}\right) .
$$

Analogously,

$$
\operatorname{det} K_{j_{2}, 1}=\frac{c^{\frac{(n-1)(n-2)}{2}}}{\lambda_{2} \lambda_{3}^{2} \cdots \lambda_{n-1}^{n-2}} \prod_{\substack{j, k=1 \\ k>j, k \neq j_{2}, j \neq j_{2}}}^{n}\left(s_{k}-s_{j}\right)
$$

from which we obtain

$$
C_{1, j_{2}}^{(1)}\left(s_{1}, \ldots, s_{n}, \delta\right)=\frac{\lambda_{1} \cdots \lambda_{n}}{c^{n}} \prod_{\substack{k=1 \\ k \neq j_{2}}}^{n} \frac{1}{s_{k}-s_{j_{2}}}
$$

and finally

$$
m_{\delta}(0)=\sum_{j_{2}=1}^{n} \frac{\lambda_{1} \cdots \lambda_{n}}{c^{n}} \prod_{\substack{k=1 \\ k \neq j_{2}}}^{n} \frac{1}{s_{k}-s_{j_{2}}} \tilde{\omega}\left(s_{j_{2}}\right) .
$$

As in Corollary 3.2, it for instance follows that the discounted joint defective density function of the surplus prior to ruin and the deficit at ruin with $x=0$ is given by

$$
f\left(y_{1}, y_{2} \mid 0\right)=\sum_{j_{2}=1}^{n} \frac{\lambda_{1} \cdots \lambda_{n}}{c^{n}} \prod_{\substack{k=1 \\ k \neq j_{2}}}^{n} \frac{1}{s_{k}-s_{j_{2}}} e^{-s_{j_{2}} y_{1}} b\left(y_{1}+y_{2}\right),
$$

which is Formula (8.3) of Gerber and Shiu [16] (for further details, see Albrecher [2]). A comparison of (26) with (22) and (23) elucidates that for $n \geq 2$ the presence of strictly positive zeroes $s_{j}$ distorts the symmetry of the classical model between the distribution of the surplus prior to ruin and of the deficit at ruin for $x=0$.

A general expression for $\tilde{m}_{\delta}(s)$ in this model can be obtained by evaluating the first row of the numerator in (15): 
Lemma 6.2. The first row of the vector $A_{\delta, a d j}(s) \vec{m}_{\delta}(0)$ is given by

$$
\left(A_{\delta, a d j}(s) \vec{m}_{\delta}(0)\right)_{1}=\frac{(-1)^{n+1} \lambda_{1} \cdots \lambda_{n}}{c} \sum_{j=1}^{n} \tilde{\omega}\left(s_{j}\right) \prod_{\substack{k=1 \\ k \neq j}}^{n} \frac{s-s_{k}}{s_{j}-s_{k}} .
$$

Proof: The first row of the adjunct matrix $A_{\delta, a d j}(s)$ of $A_{\delta}(s)$ is given by

$$
\left(A_{\delta, a d j}(s)\right)_{1}=\left(\prod_{i=2}^{n}\left(c s-\delta-\lambda_{i}\right),-\lambda_{1} \prod_{i=3}^{n}\left(c s-\delta-\lambda_{i}\right), \lambda_{1} \lambda_{2} \prod_{i=4}^{n}\left(c s-\delta-\lambda_{i}\right), \ldots,(-1)^{n+1} \lambda_{1} \cdots \lambda_{n-1}\right) .
$$

In view of (12), we have

$$
\vec{m}_{\delta}(0)=\frac{\lambda_{n}}{c \operatorname{det} K}\left(\begin{array}{c}
\operatorname{det} K_{1,1} \tilde{\omega}\left(s_{1}\right)-\operatorname{det} K_{2,1} \tilde{\omega}\left(s_{2}\right)+\ldots+(-1)^{n+1} \operatorname{det} K_{n, 1} \tilde{\omega}\left(s_{n}\right) \\
-\operatorname{det} K_{1,2} \tilde{\omega}\left(s_{1}\right)+\operatorname{det} K_{2,2} \tilde{\omega}\left(s_{2}\right)+\ldots+(-1)^{n+2} \operatorname{det} K_{n, 2} \tilde{\omega}\left(s_{n}\right) \\
\vdots \\
(-1)^{n+1} \operatorname{det} K_{1, n} \tilde{\omega}\left(s_{1}\right)+(-1)^{n+2} \operatorname{det} K_{2, n} \tilde{\omega}\left(s_{2}\right)+\ldots+\operatorname{det} K_{n, n} \tilde{\omega}\left(s_{n}\right)
\end{array}\right) .
$$

Now, collecting all coefficients of $\tilde{\omega}\left(s_{j}\right)(j=1, \ldots, n)$ in $\left(A_{\delta, a d j}(s) \vec{m}_{\delta}(0)\right)_{1}$, we obtain

$$
\begin{aligned}
& \frac{(-1)^{j+1} \lambda_{n}}{c \operatorname{det} K}\left(\prod_{i=2}^{n}\left(c s-\delta-\lambda_{i}\right) \operatorname{det} K_{j, 1}+\lambda_{1} \prod_{i=3}^{n}\left(c s-\delta-\lambda_{i}\right) \operatorname{det} K_{j, 2}\right. \\
&\left.+\ldots+\lambda_{1} \cdots \lambda_{n-1} \operatorname{det} K_{j, n}\right) \\
&= \frac{(-1)^{j+1} \lambda_{1} \cdots \lambda_{n}}{c \operatorname{det} K}\left((-1)^{n-1} \prod_{i=2}^{n} \frac{\lambda_{i}+\delta-c s}{\lambda_{i-1}} \operatorname{det} K_{j, 1}+(-1)^{n-2} \prod_{i=3}^{n} \frac{\lambda_{i}+\delta-c s}{\lambda_{i-1}} \operatorname{det} K_{j, 2}\right. \\
&\left.+\ldots+\operatorname{det} K_{j, n}\right) \\
&= \frac{(-1)^{n-1} \lambda_{1} \cdots \lambda_{n}}{c \operatorname{det} K}(-1)^{j+1} \prod_{i=2}^{n} \frac{\lambda_{i}+\delta-c s}{\lambda_{i-1}} \operatorname{det} K_{j, 1}+(-1)^{j+2} \prod_{i=3}^{n} \frac{\lambda_{i}+\delta-c s}{\lambda_{i-1}} \operatorname{det} K_{j, 2} \\
&\left.+\ldots+(-1)^{j+n} \operatorname{det} K_{j, n}\right) .
\end{aligned}
$$

But the last term in brackets is just the determinant of a matrix $K_{j}^{*}$, which is the matrix $K$ with entries $s$ instead of $s_{j}$. Hence (28) follows from $\frac{\operatorname{det} K_{j}^{*}}{\operatorname{det} K}=\prod_{\substack{k=1 \\ k \neq j}}^{n} \frac{s-s_{k}}{s_{j}-s_{k}} . \square$ Since $\left(A_{\delta, a d j}(s) \Lambda P \overrightarrow{\tilde{\omega}}(s)\right)_{1}=(-1)^{n+1} \lambda_{1} \cdots \lambda_{n} \tilde{\omega}(s)$, (15) now implies that

$$
\tilde{m}_{\delta}(s)=\frac{\tilde{\omega}(s)-\sum_{j_{2}=1}^{n} \tilde{\omega}\left(s_{j_{2}}\right) \prod_{\substack{k=1 \\ k \neq j_{2}}}^{n} \frac{s-s_{k}}{s_{j_{2}}-s_{k}}}{\prod_{j=1}^{n}\left(1+\frac{\delta-c s}{\lambda_{j}}\right)-\tilde{b}(s)},
$$


which is Equation (7.3) of Gerber and Shiu [16].

If the LST of the claim size distribution $B$ exists in a neighborhood of the origin, then (16) yields

$$
\lim _{u \rightarrow \infty} e^{R_{\delta} u} m_{\delta}(u)=\frac{\sum_{j_{2}=1}^{n} \tilde{\omega}\left(s_{j_{2}}\right) \prod_{\substack{k=1 \\ k \neq j_{2}}}^{n} \frac{-R_{\delta}-s_{k}}{s_{j_{2}}-s_{k}}-\tilde{\omega}\left(-R_{\delta}\right)}{\sum_{j=1}^{n} \frac{c}{\lambda_{j}} \prod_{\substack{k=1 \\ k \neq j}}^{n}\left(1+\frac{\delta+c R_{\delta}}{\lambda_{k}}\right)+\tilde{b}^{\prime}\left(-R_{\delta}\right)},
$$

where $-R_{\delta}$ is the largest negative zero of $\operatorname{det} A_{\delta}=0$. This formula generalizes Equation (24) of Li and Garrido [20].

Finally, in line with the approach leading to (21), one can provide an alternative proof of Identity (3.4) of Dickson and Drekic [12]:

Proposition 6.3. The joint (defective) distribution function of the surplus prior to ruin and the deficit at ruin in the Sparre Andersen model with generalized Erlang interclaim times is given by

$$
f\left(y_{1}, y_{2} \mid x\right)=\frac{\lambda_{1} \cdots \lambda_{n} b\left(y_{1}+y_{2}\right)}{c^{n} \phi(0)} \sum_{j_{2}=1}^{n}\left(\prod_{\substack{k=1 \\ k \neq j_{2}}}^{n} \frac{1}{s_{k}-s_{j_{2}}}\right) e^{-s_{j_{2}}\left(y_{1}-x\right)} \int_{\max \left\{0, x-y_{1}\right\}}^{x} e^{-s_{j_{2}} z} d \phi(z),
$$

where $\phi(x)=1-\psi(x)$ is the survival probability for initial capital $x$.

Proof: For $\delta=0$ and $w \equiv 1$ we have from (29)

$$
\tilde{\psi}(s)=\frac{\frac{1-\tilde{b}(s)}{s}-\sum_{j_{2}=1}^{n} \frac{1-\tilde{b}\left(s_{j_{2}}\right)}{s_{j_{2}}} \prod_{\substack{k=1 \\ k \neq j_{2}}}^{n} \frac{s-s_{k}}{s_{j_{2}}-s_{k}}}{\prod_{j=1}^{n}\left(1-\frac{c s}{\lambda_{j}}\right)-\tilde{b}(s)} .
$$

In terms of the survival probability, this implies

$$
\tilde{\phi}(s)=\frac{1}{s}-\tilde{\psi}(s)=\frac{\prod_{j=1}^{n}\left(1-\frac{c s}{\lambda_{j}}\right)-1+s \sum_{j_{2}=1}^{n} \frac{1-\tilde{b}\left(s_{j_{2}}\right)}{s_{j_{2}}} \prod_{\substack{k=1 \\ k \neq j_{2}}}^{n} \frac{s-s_{k}}{s_{j_{2}}-s_{k}}}{s\left(\prod_{j=1}^{n}\left(1-\frac{c s}{\lambda_{j}}\right)-\tilde{b}(s)\right)} .
$$

Obviously the numerator is a polynomial in $s$ of degree $n$. But $\tilde{\phi}(s)$ is an analytic function for $\operatorname{Re}(s)>0$ and has a simple pole at $s=0$, so that the numerator has to have the zeroes $s_{1}, \ldots, s_{n}$ (since for $\delta=0$ we have $s_{1}=0$, the latter is a zero of multiplicity 2 in the above denominator). One can deduce that the numerator is of the form $\beta \prod_{j=1}^{n}\left(s-s_{j}\right)$ for a constant $\beta \in \mathbb{R}$. By taking the limit

$$
\phi(0)=\lim _{s \rightarrow \infty} s \tilde{\phi}(s)=\frac{(-1)^{n} \beta \lambda_{1} \cdots \lambda_{n}}{c^{n}},
$$


we obtain

$$
\tilde{\phi}(s)=\frac{(-1)^{n} c^{n} \phi(0) \prod_{j=1}^{n}\left(s-s_{j}\right)}{\lambda_{1} \cdots \lambda_{n} s\left(\prod_{j=1}^{n}\left(1-\frac{c s}{\lambda_{j}}\right)-\tilde{b}(s)\right)} .
$$

Clearly, $f\left(y_{1}, y_{2} \mid x\right)$ is obtained from the discounted penalty function for $\delta=0$ and $w$ the Dirac delta function at $x_{1}=y_{1}$ and $x_{2}=y_{2}$ (so that $\tilde{\omega}(s)=e^{-s y_{1}} b\left(y_{1}+y_{2}\right)$ ). Thus the Laplace transform of $f\left(y_{1}, y_{2} \mid x\right)$ follows from (29) to be

$$
\tilde{f}\left(y_{1}, y_{2} \mid s\right)=b\left(y_{1}+y_{2}\right) \frac{e^{-s y_{1}}-\sum_{j_{2}=1}^{n} e^{-s_{j_{2}} y_{1}} \prod_{\substack{k=1 \\ k \neq j_{2}}}^{n} \frac{s-s_{k}}{s_{j_{2}}-s_{k}}}{\prod_{j=1}^{n}\left(1-\frac{c s}{\lambda_{j}}\right)-\tilde{b}(s)} .
$$

Substituting (31) into the last equation leads to

$$
\tilde{f}\left(y_{1}, y_{2} \mid s\right)=b\left(y_{1}+y_{2}\right) \frac{(-1)^{n} \lambda_{1} \cdots \lambda_{n} s \tilde{\phi}(s)}{c^{n} \phi(0)}\left(\frac{e^{-s y_{1}}}{\prod_{j=1}^{n}\left(s-s_{j}\right)}-\sum_{j_{2}=1}^{n} \frac{e^{-s_{j_{2}} y_{1}}}{s-s_{j_{2}}} \prod_{\substack{k=1 \\ k \neq j_{2}}}^{n} \frac{1}{s_{j_{2}}-s_{k}}\right) .
$$

Using partial fractions, the first term in the brackets above can be written as

$$
\frac{e^{-s y_{1}}}{\prod_{j=1}^{n}\left(s-s_{j}\right)}=\sum_{j_{2}=1}^{n} \frac{e^{-s y_{1}}}{\left(s-s_{j_{2}}\right) \prod_{\substack{k=1 \\ k \neq j_{2}}}^{n}\left(s_{j_{2}}-s_{k}\right)}
$$

and hence

$$
\tilde{f}\left(y_{1}, y_{2} \mid s\right)=b\left(y_{1}+y_{2}\right) \frac{\lambda_{1} \cdots \lambda_{n}}{c^{n} \phi(0)}\left(\sum_{j_{2}=1}^{n} s \tilde{\phi}(s) \frac{e^{-s_{j_{2}} y_{1}}-e^{-s y_{1}}}{s-s_{j_{2}}} \prod_{\substack{k=1 \\ k \neq j_{2}}}^{n} \frac{1}{s_{k}-s_{j_{2}}}\right),
$$

which is just the Laplace transform of (30).

\subsubsection{Phase-type interclaim times}

Our Markov additive process also contains the Sparre Andersen model with a phasetype interclaim time distribution and arbitrary claim size distribution. From the definition, a phase-type distribution is the lifetime of a terminating Markov process $\left\{J_{t}\right\}$ with finite state space $E$ and time homogeneous transition rates (see e.g. Asmussen [5]). In our setting, we pick out state 1 as the absorbing state of $\left\{J_{t}\right\}$ and let $p_{i j}(i, j=2, \ldots, M)$ coincide with the transition probabilities of the embedded Markov chain of $J_{t}$. A claim (with distribution $B$ ) can then only occur, if our process is in state 1 (i.e. $B_{i}$ is degenerate at 0 for $\left.i=2, \ldots, M\right)$, so that again $\tilde{B}(s)=$ $\operatorname{diag}(\tilde{b}(s), 1, \ldots, 1)$ and $\overrightarrow{\tilde{\omega}}(s)=(\tilde{\omega}(s), 0, \ldots, 0)$. Moreover, the first row entries of $P$ are given by $p_{11}=0$ and $p_{1 j}=\alpha_{j}$, where $\vec{\alpha}=\left(\alpha_{2}, \ldots, \alpha_{M}\right)$ is the $(M-1)$ dimensional vector of initial probabilities of $\left\{J_{t}\right\}$ and $p_{21}, \ldots, p_{M 1}$ represent the exit probabilities of $\left\{J_{t}\right\}$ into the absorbing state 1. If our Markov additive process $R(t)$ 
is in state 1 and a claim has occurred, it immediately jumps to one of the other states according to the vector $\vec{\alpha}$. Thus, $R(t)$ corresponds to a renewal risk model with phase-type interclaim times, if we let $\lambda_{1} \rightarrow \infty$. For then, by taking the limit in (6) for $i=1$, we obtain the discounted penalty function

$$
m_{\delta}(x):=m_{\delta, 1}(x)=\sum_{j=2}^{M} \alpha_{j} m_{\delta, j}(x) .
$$

Going to the limit $\lambda_{1} \rightarrow \infty$, one can rewrite (7) as

$$
A_{\delta}^{P h}(s) \overrightarrow{\tilde{m}}_{\delta}(s)=c\left(0, m_{\delta, 2}(0), \ldots, m_{\delta, M}(0)\right)^{T}-\Lambda^{P h} P \overrightarrow{\tilde{\omega}}(s),
$$

where $\Lambda^{P h}=\operatorname{diag}\left(1, \lambda_{2}, \ldots, \lambda_{M}\right), A_{\delta}^{P h}(s):=\left((c s-\delta) I-\Lambda^{P h}+\Lambda^{P h} P \tilde{B}(s)\right)-$ $(c s-\delta) I \vec{e}_{1} \vec{e}_{1}^{T}$ (with $\left.\vec{e}_{1}=(1,0, \ldots, 0)^{T}\right)$, i.e.

$$
A_{\delta}^{P h}(s)=\left(\begin{array}{ccccc}
-1 & \alpha_{2} & \alpha_{3} & \cdots & \alpha_{M} \\
\lambda_{2} p_{21} \tilde{b}(s) & c s-\delta-\lambda_{2}\left(1-p_{22}\right) & \lambda_{2} p_{23} & \cdots & \lambda_{2} p_{2 M} \\
\vdots & \vdots & \ddots & \ddots & \vdots \\
\lambda_{M} p_{M 1} \tilde{b}(s) & \lambda_{M} p_{M 2} & \cdots & \cdots & c s-\delta-\lambda_{M}\left(1-p_{M M}\right)
\end{array}\right)
$$

and

$$
\overrightarrow{\tilde{m}}_{\delta}(s)=\frac{A_{\delta, a d j}^{P h}(s) \cdot\left(\begin{array}{c}
0 \\
m_{\delta, 2}(0)-\lambda_{2} p_{21} \tilde{\omega}(s) \\
\vdots \\
m_{\delta, M}(0)-\lambda_{M} p_{M 1} \tilde{\omega}(s)
\end{array}\right)}{\operatorname{det} A_{\delta}^{P h}(s)}
$$

where $A_{\delta, a d j}^{P h}(s)$ is the adjugate matrix of $A_{\delta}^{P h}(s)$.

The equation $\operatorname{det} A_{\delta}^{P h}(s)=0$ has exactly $M-1$ solutions $s_{1}, \ldots, s_{M-1}$ in the right half-plane (which, for simplicity, we assume to be distinct). Thus, one can determine the unknown quantities $m_{\delta, j}(0)(j=2, \ldots, M)$ by (11) (with the obvious adaptation) and (12). Finally, $m_{\delta}(0)$ then follows from (33).

However, in the concrete situation, $m_{\delta}(0)$ can also be obtained more directly from (34): A careful analysis of the structure of $A_{\delta, a d j}^{P h}(s)$ reveals that

$$
\tilde{m}_{\delta}(s)=\frac{q_{\delta}(s)-g_{\delta}(s) \tilde{\omega}(s)}{\operatorname{det} A_{\delta}^{P h}(s)},
$$

where $q_{\delta}(s)$ is a polynomial in $s$ of degree $M-2$, the coefficients of which contain the unknown quantities $m_{\delta, j}(0)(j=2, \ldots, M)$. In addition, $g_{\delta}(s)$ is explicitly given by

$$
g_{\delta}(s)=\operatorname{det}\left((c s-\delta) I-\Lambda^{P h}+\Lambda^{P h} P\right)+{ }^{(1,1)} A_{\delta}^{P h}(s),
$$

with ${ }^{(1,1)} A_{\delta}^{P h}(s)$ denoting the minor of $A_{\delta}^{P h}(s)$ w.r.t. row and column 1 . In particular, $g_{\delta}(s)$ is also a polynomial in $s$ of degree $M-2$. Since $\tilde{m}_{\delta}(s)$ is analytic in the 
positive halfplane, the zeroes $s_{1}, \ldots, s_{M-1}$ must also be zeroes of the numerator in (35). By Lagrange interpolation we thus obtain

$$
q_{\delta}(s)=\sum_{j=1}^{M-1} g_{\delta}\left(s_{i}\right) \tilde{\omega}\left(s_{i}\right) \prod_{k=1, k \neq j}^{M-1} \frac{s-s_{k}}{s_{j}-s_{k}} .
$$

Now, $m_{\delta}(0)=\lim _{s \rightarrow \infty} s \tilde{m}_{\delta}(s)$ and it just remains to determine the latter limit. Since for $s \rightarrow \infty, \tilde{b}(s) \rightarrow 0$ and $\tilde{\omega}(s)=\mathcal{O}(1 / s)$ (the latter holds for penalty functions $w$ that do not grow super-exponentially fast), we have to collect the dominating terms in the denominator and numerator of (35), i.e.

$$
q_{\delta}(s) \sim s^{M-2} \sum_{j=1}^{M-1} g_{\delta}\left(s_{i}\right) \tilde{\omega}\left(s_{i}\right) \prod_{k=1, k \neq j}^{M-1} \frac{1}{s_{j}-s_{k}}
$$

and

$$
\operatorname{det}\left((c s-\delta) I-\Lambda^{P h}+\Lambda^{P h} P\right) \sim-c^{M-1} s^{M-1} .
$$

This finally leads to

$$
m_{\delta}(0)=\lim _{s \rightarrow \infty} s \tilde{m}_{\delta}(s)=-\frac{1}{c^{M-1}} \sum_{j=1}^{M-1} g_{\delta}\left(s_{j}\right) \tilde{\omega}\left(s_{j}\right) \prod_{k=1, k \neq j}^{M-1} \frac{1}{s_{j}-s_{k}},
$$

which is Equation (20) of $\mathrm{Li}$ and Garrido [21]. For instance, let $w\left(x_{1}, x_{2}\right)$ be the Dirac delta function with respect to $x_{1}=y_{1}, x_{2}=y_{2}$ (i.e. $\tilde{\omega}(s)=e^{-s y_{1}} b\left(y_{1}+y_{2}\right)$ ). Then we obtain from (38) the (defective) joint density function of the surplus prior to ruin and the deficit at ruin

$$
f\left(y_{1}, y_{2} \mid 0\right)=(-1)^{M-1} \frac{b\left(y_{1}+y_{2}\right)}{c^{M-1}} \sum_{j=1}^{M-1} g_{\delta}\left(s_{j}\right) e^{-s_{j} y_{1}} \prod_{k=1, k \neq j}^{M-1} \frac{1}{s_{k}-s_{j}},
$$

generalizing Formula (27) and Formula (4.6) of Dickson and Drekic [12].

For arbitrary $x>0, f\left(y_{1}, y_{2} \mid x\right)$ is, from (35), given as the inverse Laplace transform of

$$
\tilde{f}\left(y_{1}, y_{2} \mid s\right)=b\left(y_{1}+y_{2}\right) \frac{\sum_{j=1}^{M-1} g_{\delta}\left(s_{i}\right) e^{-s_{i} y_{1}} \prod_{k=1, j \neq k}^{M-1} \frac{s-s_{k}}{s_{j}-s_{k}}-g_{\delta}(s) e^{-s y_{1}}}{\operatorname{det} A_{\delta}^{P h}(s)},
$$

which can be obtained explicitly whenever the claim size distribution $B$ has a rational Laplace transform. On the other hand, for arbitrary $B$ and $\delta=0$, one can derive the following generalization of (30):

Proposition 6.4. The joint (defective) distribution function of the surplus prior to ruin and the deficit at ruin in the Sparre Andersen model with phase-type interclaim times satisfies

$$
f\left(y_{1}, y_{2} \mid x\right)=-\frac{b\left(y_{1}+y_{2}\right)}{c^{M-1} \phi(0)} \sum_{j=1}^{n} g_{0}\left(s_{j}\right)\left(\prod_{\substack{k=1 \\ k \neq j}}^{n} \frac{1}{s_{k}-s_{j}}\right) e^{-s_{j}\left(y_{1}-x\right)} \int_{\max \left\{0, x-y_{1}\right\}}^{x} e^{-s_{j} z} d \phi(z) .
$$


Proof: We will proceed in a similar fashion as in Proposition 6.3. First, one observes that

$\tilde{\phi}(s)=\frac{1}{s}-\tilde{\psi}(s)=\frac{\operatorname{det} A_{0}^{P h}(s)-s \sum_{j=1}^{M-1} g_{0}\left(s_{j}\right) \frac{1-\tilde{b}\left(s_{j}\right)}{s_{j}} \prod_{k=1, j \neq k}^{M-1} \frac{s-s_{k}}{s_{j}-s_{k}}+g_{0}(s)(1-\tilde{b}(s))}{s \operatorname{det} A_{0}^{P h}(s)}$.

The polynomial $g_{0}(s)$ can also be written as

$$
g_{0}(s)=\frac{\operatorname{det} A_{0}^{P h}(s)+{ }^{(1,1)} A_{0}^{P h}(s)}{\tilde{b}(s)},
$$

so that we are left with

$$
\tilde{\phi}(s)=\frac{-{ }^{(1,1)} A_{0}^{P h}(s)-s \sum_{j=1}^{M-1} g_{0}\left(s_{j}\right) \frac{1-\tilde{b}\left(s_{j}\right)}{s_{j}} \prod_{k=1, j \neq k}^{M-1} \frac{s-s_{k}}{s_{j}-s_{k}}+g_{0}(s)}{s \operatorname{det} A_{0}^{P h}(s)},
$$

the numerator of which is again a polynomial in $s$ of degree $M-1$. As in the proof of Proposition 6.3, it follows by analyticity arguments that

$$
\tilde{\phi}(s)=\frac{-c^{M-1} \phi(0) \prod_{j=1}^{n}\left(s-s_{j}\right)}{s \operatorname{det} A_{0}^{P h}(s)} .
$$

Now, the latter equation can be substituted in (39) which gives

$$
\tilde{f}\left(y_{1}, y_{2} \mid s\right)=\frac{b\left(y_{1}+y_{2}\right) s \tilde{\phi}(s)}{c^{M-1} \phi(0)}\left(\frac{g_{0}(s) e^{-s y_{1}}}{\prod_{k=1}^{M-1}\left(s-s_{k}\right)}-\sum_{j=1}^{M-1} \frac{g_{0}\left(s_{j}\right) e^{-s_{j} y_{1}}}{s-s_{j}} \prod_{k=1, k \neq j}^{M-1} \frac{1}{s_{j}-s_{k}}\right) .
$$

By partial fractions, we have

$$
\frac{g_{0}(s)}{\prod_{k=1}^{M-1}\left(s-s_{k}\right)}=\sum_{j=1}^{M-1} \frac{g_{0}\left(s_{j}\right)}{\prod_{k=1, k \neq j}^{M-1}\left(s_{j}-s_{k}\right)} \frac{1}{s-s_{j}}
$$

and hence

$$
\tilde{f}\left(y_{1}, y_{2} \mid s\right)=\frac{b\left(y_{1}+y_{2}\right)}{c^{M-1} \phi(0)} \sum_{j=1}^{M-1} g_{0}\left(s_{j}\right) s \tilde{\phi}(s) \frac{e^{-s y_{1}}-e^{-s_{j} y_{1}}}{s-s_{j}} \prod_{k=1, k \neq j}^{M-1} \frac{1}{s_{j}-s_{k}},
$$

which is the Laplace transform of (40).

Note that for generalized Erlang(n) interclaim times we have $g_{0}(s)=(-1)^{M+1} \lambda_{2} \cdots \lambda_{M}$ (corresponding to $(-1)^{n} \lambda_{1} \cdots \lambda_{n}$ in the notation of Subsection 6.2.1), so that indeed in this case (40) reduces to $(32)$.

Finally, our approach leads to an alternative proof of a nice identity that is due to Dickson and Drekic [12, Equ. 2.4]: 
Corollary 6.5. For $\delta=0$ we have

$$
f\left(y_{1}, y_{2} \mid x\right)=\frac{b\left(y_{1}+y_{2}\right)}{\phi(0)} \int_{\max \left\{0, x-y_{1}\right\}}^{x} \frac{f\left(y_{1}-x+z \mid 0\right)}{1-B\left(y_{1}-x+z\right)} d \phi(z) .
$$

Proof: The density of the surplus prior to ruin is obtained from $m_{\delta}(x)$ for $\delta=0$ and $w$ the Dirac-delta function w.r.t. $x_{1}=y_{1}$ (and thus $\tilde{\omega}(s)=e^{-s y_{1}}\left(1-B\left(y_{1}\right)\right)$ ). From (38) we then have

$$
f\left(y_{1} \mid 0\right)=-\frac{1-B\left(y_{1}\right)}{c^{M-1}} \sum_{j=1}^{M-1} g_{0}\left(s_{j}\right) e^{-s_{j} y_{1}} \prod_{k=1, k \neq j}^{M-1} \frac{1}{s_{j}-s_{k}} .
$$

The idea is now to consider the function

$$
\frac{f\left(y_{1}-x \mid 0\right)}{1-B\left(y_{1}-x\right)} 1_{\left\{y_{1} \geq x\right\}}
$$

whose Laplace transform w.r.t. $x$ is given by

$$
-\frac{1}{c^{M-1}} \sum_{j=1}^{M-1} g_{0}\left(s_{j}\right) e^{-s_{j} y_{1}} \frac{1-e^{-\left(s-s_{j}\right) y_{1}}}{s-s_{j}} \prod_{k=1, k \neq j}^{M-1} \frac{1}{s_{j}-s_{k}} .
$$

In view of (41), the result easily follows.

\subsection{A causal dependency model}

In Albrecher and Boxma [3], a causal dependency model was introduced, where the distribution of the interclaim time depends on the actual size of the previous claim in the following way: if the claim exceeds a (possibly random) threshold $T$, then the next interclaim time is exponentially distributed with rate $\lambda_{1}$, otherwise the interclaim time is exponentially distributed with rate $\lambda_{2}$. As mentioned in the introduction, the approach introduced in this paper contains this case for the special choice $M=2$ and

$$
d B_{1}(y)=\frac{1}{\mathbb{P}(T<B)} T(y) d B(y) \quad \text { and } \quad d B_{2}(y)=\frac{1}{\mathbb{P}(T>B)}(1-T(y)) d B(y)
$$

for a generic claim size distribution $B(y)$ and a threshold variable $T$ together with the transition probabilities $p_{i 1}=\mathbb{P}(B>T)$ and $p_{i 2}=\mathbb{P}(T>B)$ for $i=1$, 2. We can thus extend the analysis of [3] considerably, since an investigation of the discounted penalty function is at hand.

For illustration, let us work out explicit expressions for a numerical example. Let $T \sim \operatorname{Exp}(2), B \sim \operatorname{Exp}(1), c=2, \lambda_{1}=3, \lambda_{2}=1$ (which is the setup of Example 3 of $[3])$, and thus

$$
P=\left(\begin{array}{ll}
2 / 3 & 1 / 3 \\
2 / 3 & 1 / 3
\end{array}\right), \quad \Lambda=\left(\begin{array}{cc}
3 & 0 \\
0 & 1
\end{array}\right)
$$




$$
\tilde{b}_{1}(s)=\frac{3}{2}\left(\frac{1}{1+s}-\frac{1}{3+s}\right), \quad \tilde{b}_{2}(s)=\frac{3}{3+s} .
$$

Assume in the sequel $\delta=0$. Then we obtain the determinant

$$
\operatorname{det} A_{0}(s)=3-8 s+4 s^{2}+\frac{6 s-3}{1+s}-\frac{4 s}{3+s}
$$

which has one zero at 0 and one positive zero $\left(s_{2}=1.226\right.$, all the other zeroes are negative), as it should be (here and later on, all numbers are rounded to their last digit). Moreover,

$$
K=\left(\begin{array}{cc}
2 / 3 & 1 \\
-0.922 & 0.388
\end{array}\right)
$$

For an arbitrary penalty function $w$, one thus obtains from Proposition 3.1

$$
\begin{aligned}
m_{0}(0)=\left(\begin{array}{l}
0.328 \\
0.781
\end{array}\right) \tilde{\omega}_{1}(0)+\left(\begin{array}{r}
0.672 \\
-0.448
\end{array}\right) \tilde{\omega}_{1}(1.226) \\
\\
\quad+\left(\begin{array}{c}
0.164 \\
0.391
\end{array}\right) \tilde{\omega}_{2}(0)+\left(\begin{array}{r}
0.336 \\
-0.224
\end{array}\right) \tilde{\omega}_{2}(1.226) .
\end{aligned}
$$

The largest negative zero of $\operatorname{det} A_{0}(s)=0$ is given by $-R_{0}=-0.065$. Consequently, it follows from (16) and (43) that

$$
\lim _{x \rightarrow \infty} e^{0.065 x} \vec{m}_{0}(x)=\vec{C}
$$

with

$$
\begin{aligned}
\vec{C}=\left(\begin{array}{l}
6.014 \\
5.555
\end{array}\right) \tilde{\omega}_{1}(-0.065)-\left(\begin{array}{l}
5.631 \\
5.202
\end{array}\right) \tilde{\omega}_{1}(0)-\left(\begin{array}{c}
0.383 \\
0.354
\end{array}\right) \tilde{\omega}_{1}(1.226) \\
+\left(\begin{array}{l}
3.007 \\
2.778
\end{array}\right) \tilde{\omega}_{2}(-0.065)-\left(\begin{array}{l}
2.815 \\
2.601
\end{array}\right) \tilde{\omega}_{2}(0)-\left(\begin{array}{c}
0.191 \\
0.177
\end{array}\right) \tilde{\omega}_{2}(1.226) .
\end{aligned}
$$

In the special case $w \equiv 1$ (where $m_{i}(x)$ is the ruin probability $\psi_{i}(x)$, given $Z_{0}=i$ ), we have

$$
\tilde{\omega}_{1}(s)=\frac{1}{s}\left(1-\frac{3 / 2}{1+s}+\frac{3 / 2}{3+s}\right), \quad \tilde{\omega}_{2}(s)=\frac{1}{s}\left(1-\frac{3}{3+s}\right)
$$

and from $(43)$ it follows that $\psi_{1}(0)=0.945, \psi_{2}(0)=0.870$. Subsequently, from (7) we then arrive at

$$
\psi_{1}(x)=0.007 e^{-3.161 x}+0.938 e^{-0.065 x}, \quad \psi_{2}(x)=0.003 e^{-3.161 x}+0.867 e^{-0.065 x},
$$

which coincides with (26) in [3]. Moreover, in this case (44) indeed reduces to $\vec{C}=(0.938,0.867)^{T}$.

Let us now choose $w\left(x_{1}, x_{2}\right)$ as the Dirac delta function with respect to $x_{1}=y_{1}, x_{2}=$ $y_{2}$ (i.e. $\left.\tilde{\omega}_{i}(s)=e^{-s y_{1}} b_{i}\left(y_{1}+y_{2}\right)\right)$. Then $m_{0, i}(x)$ is the (defective) joint density function 
$f_{i}\left(y_{1}, y_{2} \mid x\right)$ of the surplus prior to ruin and the deficit at ruin. From (43) we obtain $\vec{m}_{0}(0)$ and then from (7) and inversion of the Laplace transform

$$
\vec{f}\left(y_{1}, y_{2} \mid x\right)=e^{-y_{2}} \vec{f}\left(y_{1} \mid x\right)
$$

with

$$
\begin{gathered}
\vec{f}\left(y_{1} \mid x\right)=1_{\left\{x \leq y_{1}\right\}}\left(\begin{array}{c}
9 \\
9
\end{array}\right) e^{-y_{1}}+e^{-3.161 x}\left(\left(\begin{array}{l}
0.106 \\
0.045
\end{array}\right) e^{-2.226 y_{1}}-\left(\begin{array}{c}
0.061 \\
0.026
\end{array}\right) e^{-y_{1}}\right) \\
+e^{-0.065 x}\left(\left(\begin{array}{c}
-0.574 \\
-0.531
\end{array}\right) e^{-2.226 y_{1}}-\left(\begin{array}{c}
8.446 \\
7.802
\end{array}\right) e^{-y_{1}}\right)+1_{\left\{x \leq y_{1}\right\}} e^{1.226 x-2.226 y_{1}}\left(\begin{array}{r}
1.476 \\
-0.186
\end{array}\right) \\
+1_{\left\{x \geq y_{1}\right\}}\left(\left(\begin{array}{c}
9.020 \\
8.333
\end{array}\right) e^{-0.0645 x-0.935 y_{1}}-\left(\begin{array}{c}
0.045 \\
0.019
\end{array}\right) e^{-3.161 x+2.161 y_{1}}\right)
\end{gathered}
$$

Note that due to the lack-of-memory property of the exponential distribution, the distribution of the deficit at ruin is again exponential and independent of the surplus before ruin. Alternatively, one could have derived the above formula using $w=e^{-a x_{1}}$ (so that $m(x)$ is the Laplace transform of the surplus before ruin) and then, by virtue of (43) and (7), inverting iteratively the Laplace transform with respect to $s$ and with respect to $a$. The moments of the surplus before ruin can now be determined either from the density above or by differentiating the above mentioned Laplace transform of the surplus before ruin. For instance,

$$
\begin{gathered}
\mathbb{E}\left(R_{T_{x}}^{-} 1_{\left\{T_{x}<\infty\right\}}\right)=\left(\begin{array}{l}
1.746 \\
1.613
\end{array}\right) e^{-0.065 x}-\left(\begin{array}{c}
0.050 \\
0.021
\end{array}\right) e^{-3.161 x}-\left(\begin{array}{r}
1 \\
0.556
\end{array}\right) e^{-x} \\
\mathbb{E}\left(\left(R_{T_{x}}^{-}\right)^{2} 1_{\left\{T_{x}<\infty\right\}}\right)=\left(\begin{array}{l}
5.041 \\
4.657
\end{array}\right) e^{-0.065 x}-\left(\begin{array}{c}
0.095 \\
0.040
\end{array}\right) e^{-3.161 x}-\left(\begin{array}{r}
2 x+3.778 \\
1.111 x+2.395
\end{array}\right) e^{-x} .
\end{gathered}
$$

In Figure 1 the density function $f_{i}\left(y_{1} \mid x\right) / \psi_{i}(x)$ of the surplus prior to ruin, given it occurs, is plotted for two specific values of initial capital $x$. Figure 2 depicts both the expected value

$$
\mathbb{E}\left(R_{T_{x}}^{-} \mid T_{x}<\infty, Z_{0}=i\right)=\frac{\mathbb{E}\left(R_{T_{x}}^{-} 1_{\left\{T_{x}<\infty\right\}} \mid Z_{0}=i\right)}{\psi_{i}(x)}
$$

and the standard deviation

$$
S D_{R_{T_{x}}^{-}}=\sqrt{\mathbb{E}\left(\left(R_{T_{x}}^{-}\right)^{2} \mid T_{x}<\infty, Z_{0}=i\right)-\mathbb{E}^{2}\left(R_{T_{x}}^{-} \mid T_{x}<\infty, Z_{0}=i\right)}
$$

as a function of initial capital $x$. Note that from the analytic expressions above, we see that $\lim _{x \rightarrow \infty} \mathbb{E}\left(R_{T_{x}}^{-} \mid T_{x}<\infty\right)=1.86$ and $\lim _{x \rightarrow \infty} S D_{R_{T_{x}}^{-}}=2.32$ (this holds for both $\left.Z_{0}=1,2\right)$.

Finally, we briefly illustrate how to use the procedure of Section 5.1 to obtain moments of the time to ruin. From (17), we have for $n=1$

$$
A_{0}(s) \vec{f}_{1}(s)=\left.c \frac{\partial \vec{m}_{\delta}(0)}{\partial \delta}\right|_{\delta=0}+\overrightarrow{\tilde{\psi}}(s)
$$



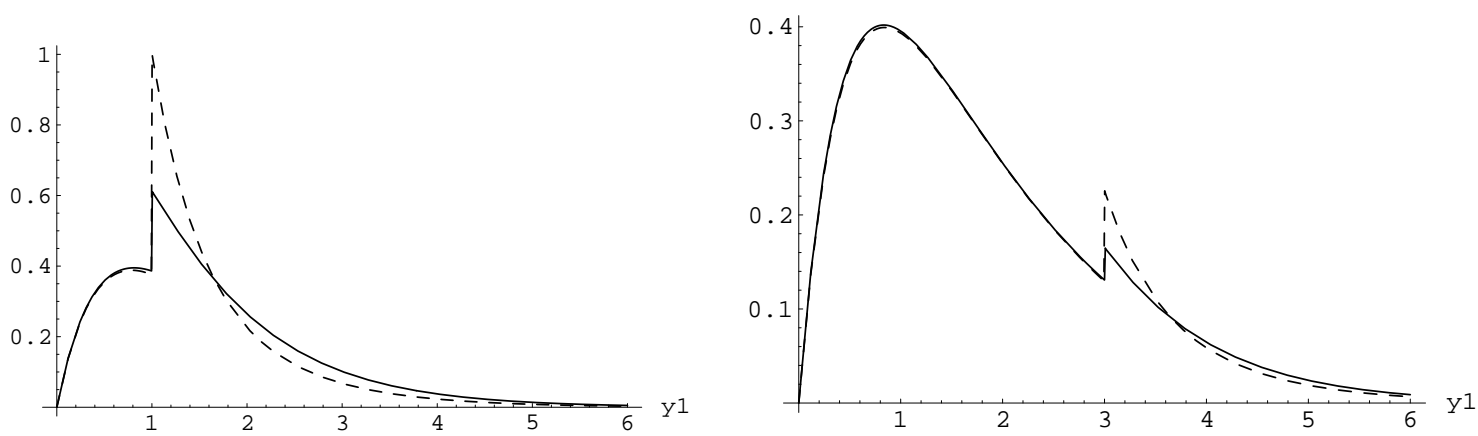

Figure 1: Density function of the surplus prior to ruin, given it occurs, for $x=1$ (left) and $x=3$ (right) (initial state $Z_{0}=1$ (dashed line) and $Z_{0}=2$ (solid line)).
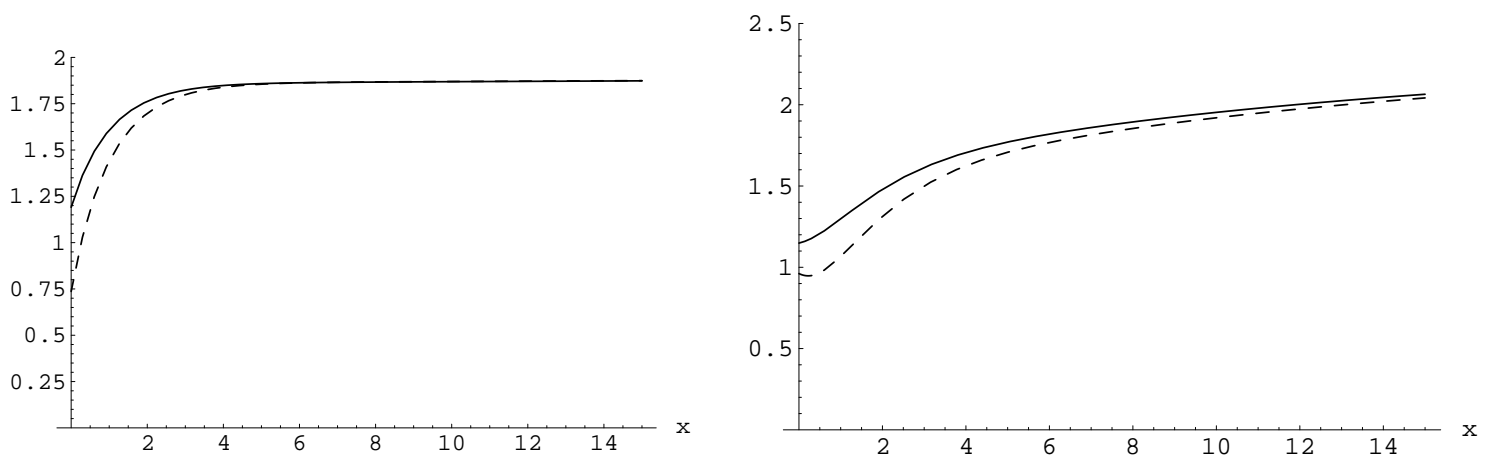

Figure 2: Expected value (left) and standard deviation (right) of the surplus prior to ruin, given it occurs (initial state $Z_{0}=1$ (dashed line) and $Z_{0}=2$ (solid line)).

By analyticity of $\vec{f}_{1}(s)$ in the right half-plane we thus obtain $\left.c \frac{\partial \vec{m}_{\delta}(0)}{\partial \delta}\right|_{\delta=0}=-\left(\begin{array}{r}7.949 \\ 17.841\end{array}\right)$ and thus, after solving (45) and inverting the Laplace transform, we obtain

$$
\mathbb{E}\left(T_{x} 1_{\left\{T_{x}<\infty\right\}}\right)=\left(\begin{array}{r}
4.330 x+4.431 \\
4 x+9.114
\end{array}\right) e^{-0.065 x}-\left(\begin{array}{c}
0.457 \\
0.193
\end{array}\right) e^{-3.161 x}
$$

Analogously, from (17),

$\mathbb{E}\left(T_{x}^{2} 1_{\left\{T_{x}<\infty\right\}}\right)=\left(\begin{array}{c}19.980 x^{2}+711.096 x+681.816 \\ 18.458 x^{2}+703.242 x+1469.25\end{array}\right) e^{-0.065 x}-\left(\begin{array}{c}75.458 \\ 32.806\end{array}\right) e^{-3.161 x}$.

Figure 3 depicts the expected value $\mathbb{E}\left(T_{x} \mid T_{x}<\infty, Z_{0}=i\right)=\frac{\mathbb{E}\left(T_{x} 1_{\left\{T_{x}<\infty\right\}} \mid Z_{0}=i\right)}{\psi_{i}(x)}$ and the standard deviation of the time to ruin, given it occurs, as a function of initial capital $x$. One clearly sees that the standard deviation of the time to ruin exceeds the expected value, so that in this dependency model it is particularly dangerous to just consider the first moment as an indicator for the riskiness of a portfolio strategy.

Acknowledgement. The authors would like to thank Clemens Heuberger for helpful advice on handling determinants. 

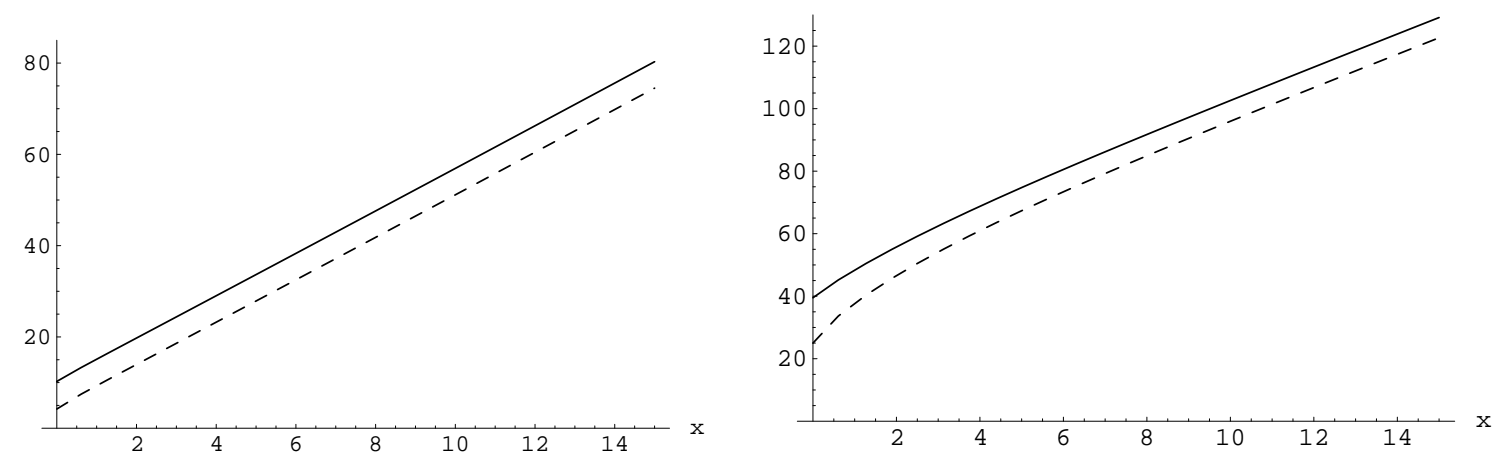

Figure 3: Expected value (left) and standard deviation (right) of the time to ruin, given it occurs (initial state $Z_{0}=1$ (dashed line) and $Z_{0}=2$ (solid line)).

\section{References}

[1] I. Adan And V. Kulkarni (2003) Single-server queue with Markov dependent interarrival and service times. Queueing Systems 45, 113-134.

[2] H. Albrecher (2005) Discussion on "The time value of ruin in a Sparre Andersen Model" by H. Gerber and E. Shiu. North American Actuarial Journal 9 (2), to appear.

[3] H. Albrecher and O.Boxma (2004) A ruin model with dependence between claim sizes and claim intervals. Insurance Math. Econom. 35 (2), 245-254.

[4] H. Albrecher and J. Teugels (2004) Exponential behavior in the presence of dependence in risk theory. EURANDOM Research Report 2004-011, TU Eindhoven.

[5] S. Asmussen (2000) Ruin Probabilities, World Scientific, Singapore, 2000.

[6] F. Avram And M. Usabel (2004) Ruin probabilities and deficit for the renewal risk model with phase-type interarrival times. Preprint.

[7] A. Badescu, L. Breuer, A. da Silva Soares, G. Latouche, M. Remiche And D. Stanford (2004) Risk processes analyzed as fluid queues. Preprint.

[8] Y. Cheng And Q. TAng (2003) Moments of the surplus before ruin and the deficit at ruin in the Erlang(2) risk process. North American Actuarial Journal, 7, 1-12.

[9] J. DE Smit (1983) The queue $G I / M / s$ with customers of different types or the queue $G I / H_{m} / s$. Adv. in Appl. Probab. 15, 392-419.

[10] D. Dickson (1992) On the distribution of the surplus prior to ruin Insurance Math. Econom. 11(3), 191-207. 
[11] D. Dickson (1998) Discussion on "On the time value of ruin" by H. Gerber and E. Shiu. North American Actuarial Journal 2(1), 74.

[12] D. Dickson And S. Drekic (2004) The joint distribution of the surplus prior to ruin and the deficit at ruin in some Sparre Andersen models. Insurance Math. Econom. 34, 97-107.

[13] D. Dickson And C. Hipp (2001) On the time to ruin for $\operatorname{Erlang}(2)$ risk processes. Insurance Math. Econom. 29, 333-344.

[14] G. Doetsch (1937) Theorie und Anwendung der Laplace-Transformation. Springer, Berlin.

[15] H. Gerber And E. Shiu (1998) On the time value of ruin. North American Actuarial Journal, 2(1), 48-78.

[16] H. Gerber And E. Shiu (2005) The time value of ruin in a Sparre Andersen Model. North American Actuarial Journal, 9(2), to appear.

[17] M. Jacobsen (2003) Martingales and the distribution of the time to ruin. Stochastic Process. Appl. 107(1), 29-51.

[18] J. Janssen and J. Reinhard (1985) Probabilités de ruine pour une classe de modèles de risque semi-Markoviens. ASTIN Bulletin 15(2), 123-134.

[19] P. Lancaster and M. Tismenetsky (1985) The Theory of Matrices (2nd ed.). Academic Press, Orlando.

[20] S. Li AND J. GarRido (2004) On ruin for the Erlang(n) risk process. Insurance Math. Econom. 34 (3), 391-408.

[21] S. Li AND J. GARRIDo (2004) On a general class of renewal risk process: Analysis of the Gerber-Shiu function. Preprint.

[22] X. Lin And G. Willmot (2000) The moments of the time to ruin, the surplus before ruin and the deficit at ruin. Insurance Math. Econom. 27, 19-44.

[23] M. Marcus And H. Minc (1964) A Survey of Matrix Theory and Matrix Inequalities. Allyn and Bacon, Boston.

[24] L. Sun AND H. YANG (2004) On the joint distributions of surplus immediately before ruin and the deficit at ruin for $\operatorname{Erlang}(2)$ risk processes. Insurance Math. Econom. 34, 121-125. 